\title{
SEARCHBreast: a new online resource to make surplus material from in vivo models of breast cancer visible and accessible to researchers
}

\author{
Bethny Morrissey ${ }^{1}$, Karen Blyth², Phil Carter ${ }^{3}$, Claude Chelala ${ }^{3}$, Louise Jones ${ }^{3}$, Ingunn Holen ${ }^{4}$ and Valerie Speirs ${ }^{1 *}$
}

Progress in understanding breast cancer biology is often underpinned by pre-clinical studies in mice. Upon completion of these studies, surplus tissues are frequently archived but often never used. There is a pressing need to make this material available so it can be shared with other research groups to answer additional scientific questions, saving time and cost by avoiding unnecessary animal models being created and, in many instances, duplicated. As well as increasing research outputs, this will reduce animal use, helping to address the 3Rs-Replacement, Refinement, and Reduction-in animal research [1].

We would like to draw attention to a new resource we have developed to help facilitate the collaborative sharing of archival tissues derived from mouse models of breast cancer [2]. Called SEARCHBreast (Sharing Experimental Animal Resources: Coordinating Holdings-Breast), this virtual resource enables researchers to access wellcharacterised excess animal materials reducing the need to initiate new in vivo studies.

The SEARCHBreast database (https://searchbreast.org) contains information on thousands of tissue samples available for immediate use following a simple online request. Samples are available from over 40 different mouse models, including transgenic, xenograft and patient-derived xenografts (PDXs). Available tissues include mammary tumours, normal mammary glands, mammary epithelial cells, and metastases (lung, liver, brain, bone). Information including genetically engineered mouse (GEM) alleles, background strain, cell lines, metastatic sites and penetrance, transplantation site, and hormone receptor status is also available. Importantly, these samples are ready for immediate use, with most available as paraffin embedded blocks or histological slides. Through bypassing the animal development pipeline

* Correspondence: v.speirs@leeds.ac.uk

${ }^{1}$ Leeds Institute of Cancer and Pathology, University of Leeds, Leeds, UK

Full list of author information is available at the end of the article which can take up to 2 years and cost upwards of US $\$ 140,000$ [2], utilising the SEARCHBreast database can save scientists both time and money. This is particularly relevant for PDX models which are costly to generate and maintain but have a growing use in many areas of breast cancer research [3]. The material remains with the owner who is under no obligation to share but may be open to collaboration. SEARCHBreast is a no-cost mediator to facilitate these collaborations. Additionally, SEARCHBreast enables scientists with little experience of in vivo work the opportunity to perform experiments under the guidance of the material owner, which could increase the quality of their research, enhancing their research output and impact [2]. By registering at https://searchbreast.org, academic researchers have free access to this innovative resource.

\section{Abbreviations}

PDX: patient-derived xenograft; SEARCHBreast: Sharing Experimental Animal Resources Coordinating Holdings-Breast.

\section{Acknowledgements}

SEARCHBreast was developed by an Infrastructure for Impact Award from NC3Rs (Grant Ref: NC/L001004/1). We thank all SEARCHBreast members for their continued support of the initiative.

\section{Authors' contributions \\ BM participated in the design of the study, management of the resource, and prepared the first draft; $P C$ participated in the design of the study and constructed the database and website; KB, CC, LJ, IH, and VS conceived of the study, secured funding and participated in its design and coordination and helped to draft the manuscript. All authors read and approved the final manuscript.}

Competing interests

The authors declare that they have no competing interests.

Consent for publication

Not applicable. 


\section{Author details}

'Leeds Institute of Cancer and Pathology, University of Leeds, Leeds, UK.

${ }^{2}$ Cancer Research UK Beatson Institute, Glasgow, UK. ${ }^{3}$ Barts Cancer Institute, London, UK. ${ }^{4}$ Academic Unit of Clinical Oncology, University of Sheffield,

Sheffield, UK.

Received: 3 May 2016 Accepted: 4 May 2016

Published online: 31 May 2016

\section{References}

1. Speirs V. Animal research: share surplus animal tissue. Nature. 2015; 522(7555):156

2. Blyth $K$, Carter P, Morrissey B, Chelala C, Jones L, Holen I, et al. SEARCHBreast: a new resource to locate and share surplus archival material from breast cancer animal models to help address the 3Rs. Breast Cancer Res Treat. 2016;156:447.

3. Whittle JR, Lewis MT, Lindeman GJ, Visvader JE. Patient-derived xenograft models of breast cancer and their predictive power. Breast Cancer Res. 2015;17:17. 